Historic, Archive Document

Do not assume content reflects current scientific knowledge, policies, or practices. 



\section{IRIS GERMANICA}

THE FLEUR DE LIS OF FRANCE

\section{PRICE LIST}

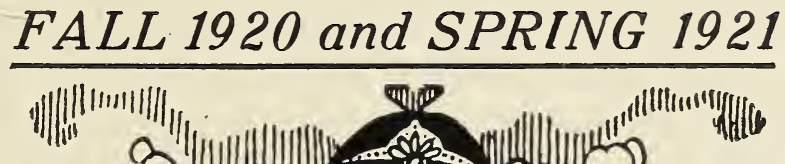

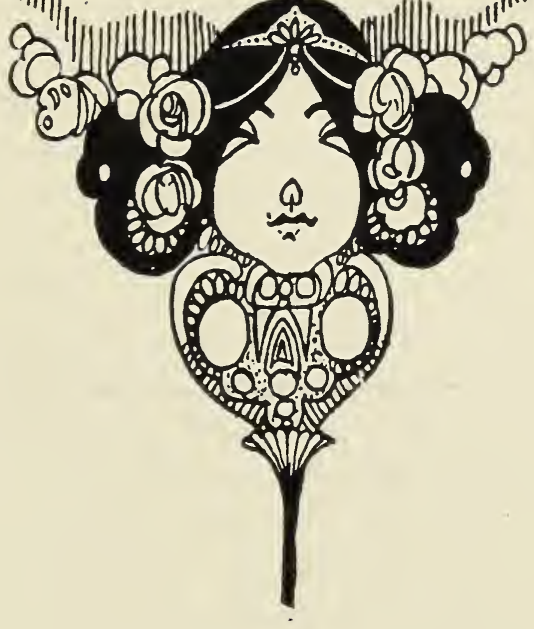

HENRY C. ECKERT

GROWER

BELLEVILLE, ILL. 
Prices quoted are for extra fine strong plants; 5 per rate of 10 ; single plants postage prepaid. Terms cash.

EACH TEN

ADNIRAL TOGO: S. \& F. white bordered lavender, same habit as Mad Chereau, extra fine 36 -inch........\$.20

$\$ 1.50$

ALBERT VICTOR: S.\& F. soft lavender blue, vigorous grower

one of the best of the Pallida section, 48-inch...... 20

BLACK PRINCE: S. Purple Lilac; F. velvety black, an early

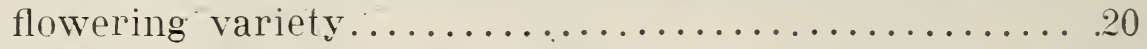

1.50

CELESTA: S. \& F. Pale azure blue, one of the most beautiful 25

CYPRIANA: S. Pale blue, F. Iong, rich purrple, resembles

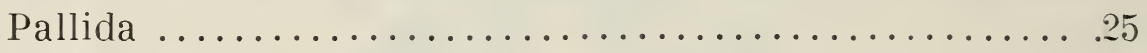

2.00

DR. BER NICE: S. Coppery bronze; F. velvety crimson, large and beautiful. Extra fine 24 -inch.................30

GERTRUDE: S. \& F. Violet blue, an early free bloomer;

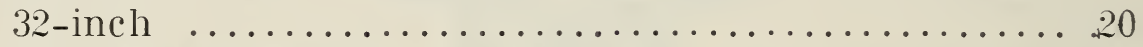

HARLEQUIN MELANOIS: S. \& F. white and blue, 32-inch; one of the most curious mottled of all irises........ .25

INGEBORG: S. \& F. pure white, large flowers, the best white in the trade; fine for cut flowers. 24 -inch........... 25

2.00

INNOCENZA: S. \& F. ivory white, crest rich golden; a fine

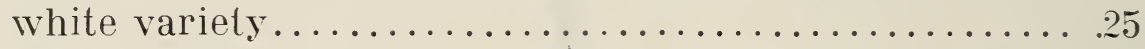

2.00

IRIS KING: S. lemon yellow; F. maroon bordered yellow; a very beautiful new variety, 34 -inch.............. 30

3.00

ISOLINE: S. lilac pink; F. purplish rose; one of the grandest varieties in cultivation, 36 -inch................ 50 4.00 JACQUESIANA: S. bright copper crimson; F. bright marroon; distinct one of the best reds, 36 -inch...........40

JOHN De WERTH: S. light mauve, F. purple, heavily vein-

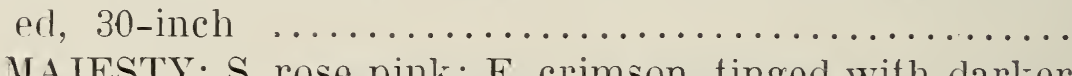

HER MAJESTY: S. rose pink; F. crimson, tinged with darker

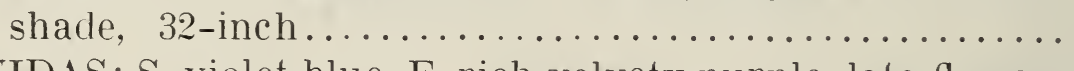

LEONIDAS: S. violet blue, F. rich velvety purple, late flower-

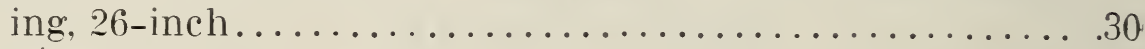
2.50 .30 .30

MACRANTHA: 'Syn. Amas) S. rich blue, F. violet, early flowering, extra fine and large, 24 -inch............30 
MAD CHEREAU: S. \& F. white frilled with a wide border of clear blue, extra fine, 34 -inch................20

MAD PACQUETTE: S. \& F. bright rosy red, distinct flowers

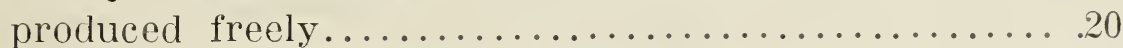

O'IHELLO: S. deep rich blue; F. dark velvety purple, very

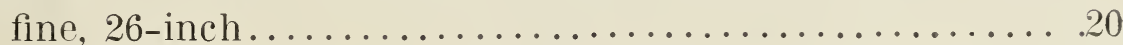

PAL. DALMATICA: S. deep lavender, F. shaded light purple,

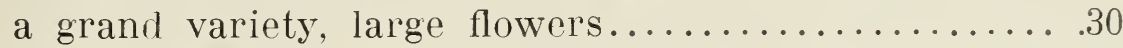

PAL. MANDRALISCOE: F. \& S. rich lavender purple, tall,

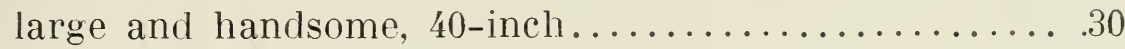

PAL. SPECIOSA: S. dark lavender shaded lighter, F. shaded

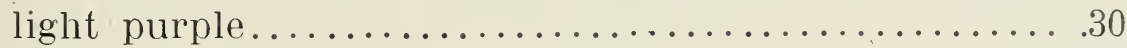

PALLIDA: S. \& F. lavender blue. This variety was bought over 50 years ago as Agathocles. The strongest grower of this section, leaves 2 inches broad, 48-inch....... 20

PARISENSIS: S. purple lilac, F. rich velvety blue, very

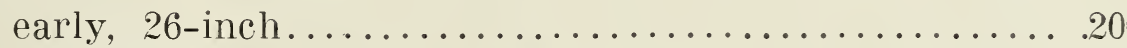

PERFECTION: S. light blue, F. dlark velvety violet, a handsome abundant bloomer, 32-inch...........25

PRINCESS VICTORIA: S. sulphur yellow, F. plum color, bordered cream, extra fine, 32 -inch...............30

QUEEN of MAY: S. \& F. soft rosy lilac shaded pink, a free

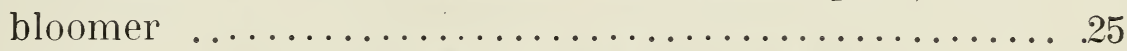

REEINNIXE: S. pure white, F. violet blue with an edge, very handsome, extra fine, 32 -inch................. 35

TAMERLANE: S. pale purple, F. deep violet purple, very large, a handsome varieety, 36-inch..............35

The following new varieties I can supply only in single plants as they did not increase any on account of the drought.

ARCHEVEQUE: S. rich purple, F. deep velvety violet; extreme rich coloring, 24-inch............... 50 CAPRICE: reddish purple, $F$. deeper purple, large and beautiful, 36 -inch..................... 50

CATERINA: Claimed to be an improved Dalmatica; a high-

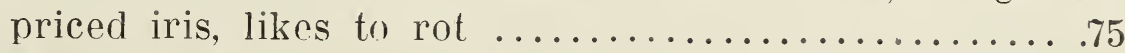
ELDORADO: S. bronze shaded yellow, F. purple bordered yellow 
LOHENGRIN: S. \& F. light rose, large and handsome,

vigorous grower...........................

MONSIGNOR: S. Lavender, F. velvety purple crimson, a large

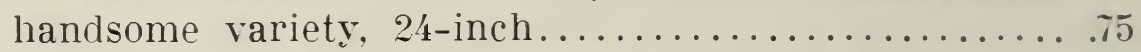

PRINCESS VICTORIA: S. \&. F. pure white, 32 -inch........ .50

QUEEN EMMA: S. \& F. clear waxy white............. .50

TWILIGHT: S. \& F. delicate eiolet purple.............50

VICTORINE: S. white; F. mottled violeet.............. .30

WYOMISSING: S. rosy white, F. deep rose shading to flesh.. .30

\section{SIBERIAN IRIS.}

The Siberian Irisis have narrow grassy foliage with beautiful flowers. Fine for cutting.

SIBERIAN BLUE $\ldots \ldots \ldots \ldots \ldots \ldots \ldots \ldots \ldots \ldots \ldots .25$

SIBERIAN GRANDIS: Violet reticulated white ..... 25

SIBERIAN ORIENTALIS: Deep blue, large for its class... 25

SIBERIAN SUPERBA: Violet blue, handsome foliage .... .25

SIBERIAN SNOW QUEEN: Ivory white flowers ........ 25

Wholesale prices per hundred will be quoted on such varieties I can supply to all growers and dealers who apply. Correspondence solicited.

HENRY C. ECKERT, Belleville, Illinois.

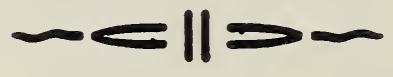

\section{THE FLOWER GROWER}

(Formerly the Modern Gladiolus Grower)

Published Monthly by Madison Cooper at Calcium, N. Y.

Subscription Price: Three Years $\$ 2.00$; One Year $\$ 1.00$ 